\title{
On Approximations by Trigonometric Polynomials of Classes of Functions Defined by Moduli of Smoothness
}

\author{
Nimete Sh. Berisha, ${ }^{1}$ Faton M. Berisha, ${ }^{2}$ Mikhail K. Potapov, ${ }^{3}$ and Marjan Dema ${ }^{4}$ \\ ${ }^{1}$ Faculty of Economics, University of Prishtina, Nëna Terezë 5, Prishtina, Kosovo \\ ${ }^{2}$ Faculty of Mathematics and Sciences, University of Prishtina, Prishtina, Kosovo \\ ${ }^{3}$ Department of Mechanics and Mathematics, Moscow State University, Moscow 117234, Russia \\ ${ }^{4}$ Faculty of Electrical and Computer Engineering, University of Prishtina, Prishtina, Kosovo \\ Correspondence should be addressed to Faton M. Berisha; faton.berisha@uni-pr.edu
}

Received 24 November 2016; Accepted 19 January 2017; Published 21 March 2017

Academic Editor: Alberto Fiorenza

Copyright (C) 2017 Nimete Sh. Berisha et al. This is an open access article distributed under the Creative Commons Attribution License, which permits unrestricted use, distribution, and reproduction in any medium, provided the original work is properly cited.

In this paper, we give a characterization of Nikol'skiǐ-Besov type classes of functions, given by integral representations of moduli of smoothness, in terms of series over the moduli of smoothness. Also, necessary and sufficient conditions in terms of monotone or lacunary Fourier coefficients for a function to belong to such a class are given. In order to prove our results, we make use of certain recent reverse Copson-type and Leindler-type inequalities.

\section{Introduction}

Let $f \in L_{p}[0,2 \pi], 1<p<\infty$, be a $2 \pi$-periodic function. We say that the function $f$ has monotone Fourier coefficients if it has a cosine Fourier series with

$$
f(x) \sim \sum_{n=0}^{\infty} a_{n} \cos n x, \quad a_{n} \downarrow 0 .
$$

We say that the function $f$ has lacunary Fourier coefficients if

$$
f(x) \sim \sum_{\nu=1}^{\infty} \lambda_{\nu} \cos \nu x
$$

where

$$
\lambda_{\nu}= \begin{cases}a_{\mu} \geq 0 & \text { for } \nu=2^{\mu} \\ 0 & \text { for } \nu \neq 2^{\mu}\end{cases}
$$

that is,

$$
f(x) \sim \sum_{\mu=0}^{\infty} a_{\mu} \cos 2^{\mu} x, \quad a_{\mu} \geq 0 .
$$

By $\omega_{k}(f, t)_{p}$ we denote the modulus of smoothness of order $k$ in $L_{p}$ metrics of a function $f \in L_{p}, 1<p<\infty$ :

$$
\omega_{k}(f, t)_{p}=\sup _{|h| \leq t}\left\|\Delta_{h}^{k} f\right\|_{p}
$$

where

$$
\Delta_{h}^{k} f(x)=\sum_{\nu=0}^{k}(-1)^{k-v}\left(\begin{array}{l}
k \\
v
\end{array}\right) f(x+\nu h)
$$

is the $k$ th-order shift operator.

By $E_{n}(f)_{p}$ we denote the best approximation in $L_{p}$ metrics of a function $f \in L_{p}, 1<p<\infty$, by means of trigonometric polynomials whose degree is not greater than $n-1$; that is,

$$
E_{n}(f)_{p}=\inf _{T_{n-1}}\left\|f-T_{n-1}\right\|_{p},
$$

where $T_{n-1}=\sum_{\nu=0}^{n-1}\left(\alpha_{\nu} \cos \nu x+\beta_{\nu} \sin \nu x\right)$ and $\alpha_{\nu}$ and $\beta_{\nu}$ are arbitrary real numbers.

We say that a $2 \pi$-periodic function $f$ belongs to the Nikol'skiü-Besov class $N(p, \theta, r, \lambda, \varphi), 1<p<\infty$, if the following conditions are satisfied:

(1) $f \in L_{p}[0,2 \pi]$. 
(2) Numbers $\theta, r$, and $\lambda$ belong to the interval $(0, \infty)$, and $k$ is an integer satisfying $k>r+\lambda$.

(3) The following inequality holds true:

$$
\begin{aligned}
& \left(\int_{0}^{\delta} t^{-r \theta-1} \omega_{k}(f, t)_{p}^{\theta} d t\right. \\
& \left.\quad+\delta^{\lambda \theta} \int_{\delta}^{1} t^{-(r+\lambda) \theta-1} \omega_{k}(f, t)_{p}^{\theta} d t\right)^{1 / \theta} \leq C \varphi(\delta),
\end{aligned}
$$

while the function $\varphi$ satisfies the following conditions:

(4) $\varphi$ is a nonnegative continuous function on $(0,1)$ and $\varphi \neq 0$.

(5) For every $\delta_{1}$ and $\delta_{2}$, where $0 \leq \delta_{1} \leq \delta_{2} \leq 1, \varphi\left(\delta_{1}\right) \leq$ $C_{1} \varphi\left(\delta_{2}\right)$ holds.

(6) For every $\delta$, where $0 \leq \delta \leq 1 / 2, \varphi(2 \delta) \leq C_{2} \varphi(\delta)$ holds.

Constants (without mentioning it explicitly, we will consider all the constants positive) $C, C_{1}$, and $C_{2}$ do not depend on $\delta_{1}$, $\delta_{2}$, and $\delta$.

A more detailed approach to the classes $N(p, \theta, r, \lambda, \varphi)$ is given in [1, 2] (see also [3]). In the paper, we give a characterization of $N(p, \theta, r, \lambda, \varphi)$ classes of functions in terms of series over their moduli of smoothness. Then we give the necessary and sufficient conditions in terms of monotone or lacunary Fourier coefficients for a function $f \in L_{p}[0,2 \pi]$ to belong to a class $N(p, \theta, r, \lambda, \varphi)$. In the process of proving the results, we make use of certain recent reverse $l_{p}$-type inequalities [4], closely related to Copson's and Leindler's inequalities.

Finally, by making use of our results, we construct an example of a function having a lacunary Fourier series, which shows that $N(p, \theta, r, \lambda, \varphi)$ classes are properly embedded between the appropriate Nikol'skii classes and Besov classes.

\section{Statement of Results}

Now we formulate our results.

Theorem 1. A function $f$ belongs to the class $N(p, \theta, r, \lambda, \varphi)$ if and only if (here and below we assume that the parameters $\theta, r, \lambda$, and $k$ satisfy condition (2) and the function $\varphi$ satisfies conditions (4)-(6) of the definition of the class $N(p, \theta, r, \lambda, \varphi)$ )

$$
\begin{aligned}
& \left(\sum_{\nu=n+1}^{\infty} \omega_{k}\left(f, \frac{1}{\nu}\right)_{p}^{\theta} \nu^{r \theta-1}\right. \\
& \left.+n^{-\lambda \theta} \sum_{\nu=1}^{n} \omega_{k}\left(f, \frac{1}{v}\right)_{p}^{\theta} \nu^{(r+\lambda) \theta-1}\right)^{1 / \theta} \leq C \varphi\left(\frac{1}{n}\right),
\end{aligned}
$$

where constant $C$ does not depend on $n$.

Theorem 2. For a function $f \in L_{p}[0,2 \pi], 1<p<\infty$, such that

$$
f(x) \sim \sum_{\nu=1}^{\infty} a_{\nu} \cos \nu x, \quad a_{\nu} \downarrow 0,
$$

to belong to the class $N(p, \theta, r, \lambda, \varphi)$, it is necessary and sufficient that its Fourier coefficients satisfy the condition

$$
\begin{aligned}
& \left(\sum_{\nu=n+1}^{\infty} a_{\nu}^{\theta} \nu^{r \theta+\theta-\theta / p-1}+n^{-\lambda \theta} \sum_{\nu=1}^{n} a_{\nu}^{\theta} \nu^{r \theta+\lambda \theta+\theta-\theta / p-1}\right)^{1 / \theta} \\
& \quad \leq C \varphi\left(\frac{1}{n}\right),
\end{aligned}
$$

where constant $C$ does not depend on $n$.

Regarding Theorem 1, a very interesting open question on its analogue for functions with general monotone Fourier coefficients generalized in the sense of $[5,6]$ remains.

Corollary 3. Putting $\varphi(\delta)=\delta^{\alpha}, 0<\alpha<\lambda$, in the definition of the class $N(p, \theta, r, \lambda, \varphi)$, we obtain [1] the Nikol'skiu class $H_{p}^{r+\alpha}$. Thus Theorems 1 and 2 give the single coefficient condition

$$
a_{v} \leq \frac{C}{v^{r+\alpha+1-1 / p}}
$$

for $f \in H_{p}^{r+\alpha}$, given in [7], where the function $f$ is given by (10).

Corollary 4. If $\varphi(\delta) \geq C$, then we obtain [1] the Besov class $B_{p}^{\theta r}$. Thus Theorems 1 and 2 give the necessary and sufficient condition

$$
\sum_{\nu=1}^{\infty} a_{\nu}^{\theta} v^{r \theta+\theta-\theta / p-1}<\infty
$$

for $f \in B_{p}^{\theta r}$, given in [8], where the function $f$ is given by (10).

Theorem 5. For a function $f \in L_{p}, 1<p<\infty$, such that

$$
\begin{aligned}
f(x) & \sim \sum_{\nu=1}^{\infty} \lambda_{\nu} \cos \nu x, \\
\lambda_{\nu} & = \begin{cases}a_{\mu} \geq 0 & \text { for } \nu=2^{\mu}, \\
0 & \text { for } \nu \neq 2^{\mu},\end{cases}
\end{aligned}
$$

to belong to the class $N(p, \theta, r, \lambda, \varphi)$, it is necessary and sufficient that its Fourier coefficients satisfy the condition (here and below we assume that the parameters $\theta, r, \lambda$, and $k$ satisfy condition (2) and the function $\varphi$ satisfies conditions (4)-(6).)

$$
\left(\sum_{\nu=m+1}^{\infty} \lambda_{\nu}^{\theta} \nu^{r \theta}+m^{-\lambda \theta} \sum_{\nu=1}^{m} \lambda_{\nu}^{\theta} \nu^{(r+\lambda) \theta}\right)^{1 / \theta} \leq C \varphi\left(\frac{1}{m}\right),
$$

where constant $C$ does not depend on $m$.

Corollary 6. Putting $\varphi(\delta)=\delta^{\alpha}, 0<\alpha<\lambda$, in the definition of the class $N(p, \theta, r, \lambda, \varphi)$, we obtain [1] the Nikol'skiu class $H_{p}^{r+\alpha}$. Thus Theorem 5 gives the single coefficient condition

$$
a_{\mu} \leq C 2^{-\mu(r+\alpha)}
$$

for $f \in H_{p}^{r+\alpha}$, where the function $f$ is given by (14). 
Corollary 7. If $\varphi(\delta)=C$, then we obtain [1] the Besov class $B_{p}^{\theta r}$. Thus Theorem 5 gives the necessary and sufficient condition

$$
\sum_{\mu=1}^{\infty} a_{\mu}^{\theta} 2^{\mu r \theta}<\infty
$$

for $f \in B_{p}^{\theta r}$, given in [8], where the function $f$ is given by (14).

Example 8. Let

$$
f(x) \sim \sum_{\mu=0}^{\infty} a_{\mu} \cos 2^{\mu} x,
$$

where

$$
a_{\mu}=2^{-\mu r}(\mu+1)^{-(\alpha+1 / \theta)}, \quad \alpha>0 .
$$

Then, we have

$$
\begin{aligned}
C_{1} n^{-\alpha} & \leq\left(\sum_{\mu=n+1}^{\infty} a_{\mu}^{\theta} 2^{\mu r \theta}\right)^{1 / \theta} \leq C_{2} n^{-\alpha}, \\
C_{3} n^{-(\alpha+1 / \theta)} & \leq\left(2^{-n \lambda \theta} \sum_{\mu=0}^{n} a_{\mu}^{\theta} 2^{\mu(r+\lambda) \theta}\right)^{1 / \theta} \leq C_{4} n^{-(\alpha+1 / \theta)},
\end{aligned}
$$

thus implying (see the proof of Theorem 5) that $f \in$ $N(p, \theta, r, \lambda, \varphi)$ for $\varphi(\delta)=(\ln (1 / \delta))^{-\alpha}$. This means that classes $N$ are classes of embedding between classes $H$ and $B$.

\section{Auxiliary Statements}

In order to establish our results, we use the following lemmas.

Lemma 9. Let $0<\alpha<\beta<\infty$ and $a_{v} \geq 0$. The following inequality holds true:

$$
\left(\sum_{\nu=1}^{n} a_{\nu}^{\beta}\right)^{1 / \beta} \leq\left(\sum_{\nu=1}^{n} a_{\nu}^{\alpha}\right)^{1 / \alpha} .
$$

Proof of the lemma is due to Jensen [9, p. 43].

Lemma 10. Let $\left\{a_{\gamma}\right\}_{\nu=1}^{\infty}$ be a sequence of nonnegative numbers, let $\alpha>0$, let $\lambda$ be a real number, and let $m$ and $n$ be positive integers such that $m<n$. Then

(1) for $1 \leq p<\infty$, the following equalities hold:

$$
\begin{aligned}
& \sum_{\mu=m}^{n} \mu^{\alpha-1}\left(\sum_{\nu=\mu}^{n} a_{\nu} \nu^{\lambda}\right)^{p} \leq C_{1} \sum_{\mu=m}^{n} \mu^{\alpha-1}\left(a_{\mu} \mu^{\lambda+1}\right)^{p}, \\
& \sum_{\mu=m}^{n} \mu^{-\alpha-1}\left(\sum_{\nu=m}^{\mu} a_{\nu} \nu^{\lambda}\right)^{p} \leq C_{2} \sum_{\mu=m}^{n} \mu^{-\alpha-1}\left(a_{\mu} \mu^{\lambda+1}\right)^{p} ;
\end{aligned}
$$

(2) for $0<p \leq 1$, the following equalities hold:

$$
\begin{gathered}
\sum_{\mu=m}^{n} \mu^{\alpha-1}\left(\sum_{\nu=\mu}^{n} a_{\nu} \nu^{\lambda}\right)^{p} \geq C_{3} \sum_{\mu=m}^{n} \mu^{\alpha-1}\left(a_{\mu} \mu^{\lambda+1}\right)^{p}, \\
\sum_{\mu=m}^{n} \mu^{-\alpha-1}\left(\sum_{\nu=m}^{\mu} a_{\nu} \nu^{\lambda}\right)^{p} \geq C_{4} \sum_{\mu=m}^{n} \mu^{-\alpha-1}\left(a_{\mu} \mu^{\lambda+1}\right)^{p},
\end{gathered}
$$

where constants $C_{1}, C_{2}, C_{3}$, and $C_{4}$ depend only on numbers $\alpha, \lambda$, and $p$ and do not depend on $m$ and $n$ as well as on the sequence $\left\{a_{\nu}\right\}_{\gamma=1}^{\infty}$.

Proof of the lemma is given in [9, p. 308].

Lemmas 11 and 12 state certain $l_{p}$-type inequalities which are reversed to the ones given in Lemma 10 and closely related to Copson's and Leindler's inequalities (see, e.g., [10-13]).

We write $a_{v} \downarrow$ if $\left\{a_{\gamma}\right\}_{\gamma=1}^{\infty}$ is a monotone-decreasing sequence of nonnegative numbers, that is, if $a_{v} \geq a_{v+1} \geq$ $0(\nu=1,2, \ldots)$.

Lemma 11. Let $a_{v} \downarrow$, let $\alpha>0$, let $\lambda$ be a real number, and let $m$ and $n$ be positive integers. Then

(1) for $1 \leq p<\infty$ and $n \geq 16 m$, the following equalities hold:

$$
\begin{gathered}
\sum_{\mu=m}^{n} \mu^{\alpha-1}\left(\sum_{\nu=\mu}^{n} a_{\nu} \nu^{\lambda}\right)^{p} \geq C_{1} \sum_{\mu=8 m}^{n} \mu^{\alpha-1}\left(a_{\mu} \mu^{\lambda+1}\right)^{p}, \\
\sum_{\mu=m}^{n} \mu^{-\alpha-1}\left(\sum_{\nu=m}^{\mu} a_{\nu} \nu^{\lambda}\right)^{p} \geq C_{2} \sum_{\mu=4 m}^{n} \mu^{-\alpha-1}\left(a_{\mu} \mu^{\lambda+1}\right)^{p} ;
\end{gathered}
$$

(2) for $0<p \leq 1$ and $n \geq 4 m$, the following equalities hold:

$$
\begin{gathered}
\sum_{\mu=4 m}^{n} \mu^{\alpha-1}\left(\sum_{\nu=\mu}^{n} a_{\nu} \nu^{\lambda}\right)^{p} \leq C_{3} \sum_{\mu=m}^{n} \mu^{\alpha-1}\left(a_{\mu} \mu^{\lambda+1}\right)^{p}, \\
\sum_{\mu=4 m}^{n} \mu^{-\alpha-1}\left(\sum_{\nu=4 m}^{\mu} a_{\nu} \nu^{\lambda}\right)^{p} \leq C_{4} \sum_{\mu=m}^{n} \mu^{-\alpha-1}\left(a_{\mu} \mu^{\lambda+1}\right)^{p},
\end{gathered}
$$

where constants $C_{1}, C_{2}, C_{3}$, and $C_{4}$ depend only on numbers $\alpha, \lambda$, and $p$ and do not depend on $m$ and $n$ as well as on the sequence $\left\{a_{\nu}\right\}_{\nu=1}^{\infty}$.

Proof of the lemma is given in [4].

Lemma 12. Let $a_{\gamma} \downarrow$, let $\alpha>0$, let $\lambda$ be a real number, and let $m$ and $n$ be positive integers. For $0<p<\infty$, the following inequalities hold:

$$
\begin{aligned}
& C_{1} \sum_{\mu=1}^{n} \mu^{\alpha-1}\left(a_{\mu} \mu^{\lambda+1}\right)^{p} \leq \sum_{\mu=1}^{n} \mu^{\alpha-1}\left(\sum_{\nu=\mu}^{n} a_{\nu} \nu^{\lambda}\right)^{p} \\
& \leq C_{2} \sum_{\mu=1}^{n} \mu^{\alpha-1}\left(a_{\mu} \mu^{\lambda+1}\right)^{p}, \\
& C_{3} \sum_{\mu=1}^{n} \mu^{-\alpha-1}\left(a_{\mu} \mu^{\lambda+1}\right)^{p} \leq \sum_{\mu=1}^{n} \mu^{-\alpha-1}\left(\sum_{\nu=1}^{\mu} a_{\nu} \nu^{\lambda}\right)^{p} \\
& \leq C_{4} \sum_{\mu=1}^{n} \mu^{-\alpha-1}\left(a_{\mu} \mu^{\lambda+1}\right)^{p},
\end{aligned}
$$

where constants $C_{1}, C_{2}, C_{3}$, and $C_{4}$ depend only on numbers $\alpha, \lambda$, and $p$ and do not depend on $m$ and $n$ as well as on the sequence $\left\{a_{\nu}\right\}_{\nu=1}^{\infty}$. 
The lemma is also proven in [4].

Lemma 13. Let $f \in L_{p}[0,2 \pi]$ for fixed $p$ from the interval $1<p<\infty$ and let

$$
f(x) \sim \sum_{\nu=1}^{\infty} a_{\nu} \cos \nu x, \quad a_{\nu} \downarrow 0 .
$$

The following inequalities hold:

$$
\begin{aligned}
C_{1} & \frac{1}{n^{k}}\left(\sum_{\nu=1}^{n} a_{\nu}^{p} \nu^{(k+1) p-2}\right)^{1 / p}+\left(\sum_{\nu=n+1}^{\infty} a_{\nu}^{p} \nu^{p-2}\right)^{1 / p} \\
& \leq \omega_{k}\left(f, \frac{1}{n}\right)_{p} \\
& \leq C_{2} \frac{1}{n^{k}}\left(\sum_{\nu=1}^{n} a_{\nu}^{p} \nu^{(k+1) p-2}\right)^{1 / p}+\left(\sum_{\nu=n+1}^{\infty} a_{\nu}^{p} \nu^{p-2}\right)^{1 / p},
\end{aligned}
$$

where constants $C_{1}$ and $C_{2}$ do not depend on $n$ and $f$.

The lemma is proven in [8].

Lemma 14. A function $f$ belongs to the class $N(p, \theta, r, \lambda, \varphi)$ if and only if

$$
\begin{aligned}
& \left(\sum_{\mu=n+1}^{\infty} 2^{\mu r \theta} E_{2^{\mu}}(f)_{p}^{\theta}+2^{-n \lambda \theta} \sum_{\mu=0}^{n} 2^{\mu(r+\lambda) \theta} E_{2^{\mu}}(f)_{p}^{\theta}\right)^{1 / \theta} \\
& \quad \leq C \varphi\left(\frac{1}{2^{n}}\right)
\end{aligned}
$$

where constant $C$ does not depend on $n$.

Proof of the lemma is given in [1].

Lemma 15. Let $f \in L_{p}, 1<p<\infty$, and

$$
f(x) \sim \sum_{\mu=0}^{\infty} a_{\mu} \cos 2^{\mu} x, \quad a_{\mu} \geq 0 .
$$

The following inequalities hold:

$$
C_{1}\left(\sum_{\mu=0}^{\infty} a_{\mu}^{2}\right)^{1 / 2} \leq\|f\|_{p} \leq C_{2}\left(\sum_{\mu=0}^{\infty} a_{\mu}^{2}\right)^{1 / 2}
$$

where constants $C_{2}$ and $C_{1}$ do not depend on $f$.

Proof of the lemma is due to Zygmund [14, vol. I, p. 326].

Corollary 16. Lemma 15 yields the following estimate:

$$
C_{1}\left(\sum_{\mu=n}^{\infty} a_{\mu}^{2}\right)^{1 / 2} \leq E_{2^{n}}(f)_{p} \leq C_{2}\left(\sum_{\mu=n}^{\infty} a_{\mu}^{2}\right)^{1 / 2},
$$

where constants $C_{2}$ and $C_{1}$ do not depend on $n$ and $f$.

\section{Proofs}

Now we prove our results.

Proof of Theorem 1. Put

$$
\begin{aligned}
& I_{1}=\int_{0}^{1 /(n+1)} t^{-r \theta-1} \omega_{k}(f, t)_{p}^{\theta} d t, \\
& I_{2}=\int_{1 /(n+1)}^{1} t^{-(r+\lambda) \theta-1} \omega_{k}(f, t)_{p}^{\theta} d t .
\end{aligned}
$$

We have $[9$, p. 55]

$$
\begin{aligned}
I_{1} & =\int_{0}^{1 /(n+1)} t^{-r \theta-1} \omega_{k}(f, t)_{p}^{\theta} d t \\
& =\sum_{\nu=n+1}^{\infty} \int_{1 /(\nu+1)}^{1 / \nu} t^{-r \theta-1} \omega_{k}(f, t)_{p}^{\theta} d t \\
& \leq \sum_{\nu=n+1}^{\infty} \omega_{k}\left(f, \frac{1}{\nu}\right)_{p}^{\theta} \int_{1 /(v+1)}^{1 / \nu} t^{-r \theta-1} d t \\
& \leq C_{1} \sum_{\nu=n+1}^{\infty} \omega_{k}\left(f, \frac{1}{v}\right)_{p}^{\theta} v^{r \theta-1}
\end{aligned}
$$

and, taking into account properties of modulus of smoothness [15],

$$
\begin{aligned}
I_{1} & \geq \sum_{\nu=n+1}^{\infty} \omega_{k}\left(f, \frac{1}{\nu+1}\right)_{p}^{\theta} \int_{1 /(\nu+1)}^{1 / \nu} t^{-r \theta-1} d t \\
& \geq C_{2} \sum_{\nu=n+1}^{\infty} \omega_{k}\left(f, \frac{1}{\nu}\right)_{p}^{\theta} \nu^{r \theta-1} .
\end{aligned}
$$

In an analogous way, we estimate

$$
\begin{aligned}
I_{2} & \leq \sum_{\nu=1}^{n} \omega_{k}\left(f, \frac{1}{v}\right)_{p}^{\theta} \int_{1 /(v+1)}^{1 / v} t^{-(r+\lambda) \theta-1} d t \\
& \leq C_{3} \sum_{\nu=1}^{n} \omega_{k}\left(f, \frac{1}{v}\right)_{p}^{\theta} v^{(r+\lambda) \theta-1}, \\
I_{2} & \geq \sum_{\nu=1}^{n} \omega_{k}\left(f, \frac{1}{v+1}\right)_{p}^{\theta} \int_{1 /(v+1)}^{1 / \nu} t^{-(r+\lambda) \theta-1} d t \\
& \geq C_{4} \sum_{\nu=1}^{n} \omega_{k}\left(f, \frac{1}{v}\right)_{p}^{\theta} v^{(r+\lambda) \theta-1} .
\end{aligned}
$$

Let $f \in N(p, \theta, r, \lambda, \varphi)$. For a positive integer $n$, we put $\delta=$ $1 /(n+1)$. Then we have

$$
\begin{aligned}
I^{\theta} & =I_{1}+\delta^{\lambda \theta} I_{2} \geq C_{5}\left(\sum_{\nu=n+1}^{\infty} \omega_{k}\left(f, \frac{1}{v}\right)_{p}^{\theta} v^{r \theta-1}\right. \\
& \left.+n^{-\lambda \theta} \sum_{\nu=1}^{n} \omega_{k}\left(f, \frac{1}{v}\right)_{p}^{\theta} v^{(r+\lambda) \theta-1}\right) .
\end{aligned}
$$


Hence, we obtain

$$
\begin{aligned}
J= & \left(\sum_{\nu=n+1}^{\infty} \omega_{k}\left(f, \frac{1}{\nu}\right)_{p}^{\theta} \nu^{r \theta-1}\right. \\
& \left.+n^{-\lambda \theta} \sum_{\nu=1}^{n} \omega_{k}\left(f, \frac{1}{\nu}\right)_{p}^{\theta} \nu^{(r+\lambda) \theta-1}\right)^{1 / \theta} \leq C_{6} I \\
& \leq C_{7} \varphi(\delta)=C_{7} \varphi\left(\frac{1}{n+1}\right) \leq C_{8} \varphi\left(\frac{1}{n}\right),
\end{aligned}
$$

which proves inequality (9).

Now we suppose that inequality (9) holds. For $\delta \in(0,1)$, we choose the positive integer $n$ satisfying $1 /(n+1)<\delta \leq 1 / n$. Then, taking into consideration the estimates from above for $I_{1}$ and $I_{2}$, we have

$$
\begin{aligned}
I^{\theta} & =\int_{0}^{1 /(n+1)} t^{-r \theta-1} \omega_{k}(f, t)_{p}^{\theta} d t \\
& +\int_{1 /(n+1)}^{\delta} t^{-r \theta-1} \omega_{k}(f, t)_{p}^{\theta} d t \\
& +\delta^{\lambda \theta} \int_{\delta}^{1} t^{-(r+\lambda) \theta-1} \omega_{k}(f, t)_{p}^{\theta} d t \leq I_{1}+\delta^{\lambda \theta} I_{2} \\
& \leq C_{9}\left(\sum_{\nu=n+1}^{\infty} \omega_{k}\left(f, \frac{1}{v}\right)_{p}^{\theta} \nu^{r \theta-1}\right. \\
& \left.+n^{-\lambda \theta} \sum_{\nu=1}^{n} \omega_{k}\left(f, \frac{1}{v}\right)_{p}^{\theta} v^{(r+\lambda) \theta-1}\right) .
\end{aligned}
$$

Hence

$$
I \leq C_{10} J \leq C_{11} \varphi\left(\frac{1}{n}\right) \leq C_{12} \varphi\left(\frac{1}{2 n}\right) \leq C_{13} \varphi(\delta),
$$

implying that $f \in N(p, \theta, r, \lambda, \varphi)$.

Proof of Theorem 1 is completed.

Proof of Theorem 2. Theorem 1 implies that the condition $f \in$ $N(p, \theta, r, \lambda, \varphi)$ is equivalent to the condition

$$
\begin{aligned}
& \sum_{\nu=n+1}^{\infty} \omega_{k}\left(f, \frac{1}{v}\right)_{p}^{\theta} \nu^{r \theta-1}+n^{-\lambda \theta} \sum_{\nu=1}^{n} \omega_{k}\left(f, \frac{1}{\nu}\right)_{p}^{\theta} \nu^{(r+\lambda) \theta-1} \\
& \leq C_{1} \varphi\left(\frac{1}{n}\right)^{\theta},
\end{aligned}
$$

where constant $C_{1}$ does not depend on $n$. Lemma 13 yields that the last estimate is equivalent to the estimate

$$
\begin{gathered}
\sum_{\nu=n+1}^{\infty} \nu^{(r-k) \theta-1}\left(\sum_{\mu=1}^{\nu} a_{\mu}^{p} \mu^{(k+1) p-2}\right)^{\theta / p} \\
+\sum_{\nu=n+1}^{\infty} \nu^{r \theta-1}\left(\sum_{\mu=\nu}^{\infty} a_{\mu}^{p} \mu^{p-2}\right)^{\theta / p}
\end{gathered}
$$

$$
\begin{aligned}
& +n^{-\lambda \theta} \sum_{\nu=1}^{n} \nu^{(r+\lambda-k) \theta-1}\left(\sum_{\mu=1}^{\nu} a_{\mu}^{p} \mu^{(k+1) p-2}\right)^{\theta / p} \\
& +n^{-\lambda \theta} \sum_{\nu=1}^{n} \nu^{(r+\lambda) \theta-1}\left(\sum_{\mu=\nu}^{\infty} a_{\mu}^{p} \mu^{p-2}\right)^{\theta / p} \\
& \leq C_{2} \varphi\left(\frac{1}{n}\right)^{\theta}
\end{aligned}
$$

where constant $C_{2}$ does not depend on $n$. Hence, if we denote the terms on the left-hand side of the inequality by $J_{1}, J_{2}$, $J_{3}$, and $J_{4}$, respectively, then condition $f \in N(p, \theta, r, \lambda, \varphi)$ is equivalent to the condition

$$
J_{1}+J_{2}+J_{3}+J_{4} \leq C_{2} \varphi\left(\frac{1}{n}\right)^{\theta}
$$

Now we estimate the terms $J_{1}, J_{2}, J_{3}$, and $J_{4}$ from below and above by means of expression taking part in the condition of the theorem.

First we estimate $J_{1}$ and $J_{2}$ from below. We have

$$
\begin{aligned}
J_{1} & =\sum_{\nu=n+1}^{\infty} \nu^{(r-k) \theta-1}\left(\sum_{\mu=1}^{\nu} a_{\mu}^{p} \mu^{(k+1) p-2}\right)^{\theta / p} \\
& \geq \sum_{\nu=n+1}^{\infty} \nu^{-(k-r) \theta-1}\left(\sum_{\mu=n+1}^{\nu} a_{\mu}^{p} \mu^{(k+1) p-2}\right)^{\theta / p} .
\end{aligned}
$$

For $k-r>0$, making use of Lemmas 10 and 11, we obtain

$$
\begin{aligned}
J_{1} & \geq C_{3} \sum_{\nu=4(n+1)}^{\infty} v^{-(k-r) \theta-1}\left(a_{\nu}^{p} \nu^{(k+1) p-2 v}\right)^{\theta / p} \\
& =C_{3} \sum_{\nu=4(n+1)}^{\infty} a_{\nu}^{\theta} v^{r \theta+\theta-\theta / p-1} .
\end{aligned}
$$

In an analogous way, for $r \theta>0$, we get

$$
\begin{aligned}
J_{2} & =\sum_{\nu=n+1}^{\infty} \nu^{r \theta-1}\left(\sum_{\mu=\nu}^{\infty} a_{\mu}^{p} \mu^{p-2}\right)^{\theta / p} \\
& \geq C_{4} \sum_{\nu=8(n+1)}^{\infty} a_{\nu}^{\theta} \nu^{r \theta+\theta-\theta / p-1} .
\end{aligned}
$$

We estimate the term $J_{2}$ from above:

$$
\begin{aligned}
J_{2} & \leq C_{5} \sum_{\nu=[(n+1) / 4]}^{\infty} \nu^{r \theta-1}\left(a_{\nu}^{p} \nu^{p-2 \nu}\right)^{\theta / p} \\
& =C_{5} \sum_{\nu=[(n+1) / 4]}^{\infty} a_{\nu}^{\theta} \nu^{r \theta+\theta-\theta / p-1} .
\end{aligned}
$$


For $J_{1}$, we have

$$
\begin{aligned}
J_{1} & \leq C_{6}\left(\sum_{\nu=n+1}^{\infty} \nu^{-(k-r) \theta-1}\left(\sum_{\mu=n+1}^{\nu} a_{\mu}^{p} \mu^{(k+1) p-2}\right)^{\theta / p}\right. \\
& \left.+\sum_{\nu=n+1}^{\infty} \nu^{-(k-r) \theta-1}\left(\sum_{\mu=1}^{n} a_{\mu}^{p} \mu^{(k+1) p-2}\right)^{\theta / p}\right),
\end{aligned}
$$

and applying once more Lemmas 10 and 11, we obtain

$$
\begin{aligned}
J_{1} \leq & C_{7} \sum_{\nu=[(n+1) / 4]}^{\infty} a_{\nu}^{\theta} \nu^{r \theta+\theta-\theta / p-1} \\
& +n^{-(k-r) \theta}\left(\sum_{\mu=1}^{n} a_{\mu}^{p} \mu^{(k+1) p-2}\right)^{\theta / p} .
\end{aligned}
$$

Put

$$
I_{1}=n^{-(k-r) \theta} \sum_{\mu=1}^{n} a_{\mu}^{p} \mu^{(k+1) p-2} .
$$

Then, for

$$
I_{2}=I_{1} n^{(k-r) \theta},
$$

taking into account the fact that $(k+1) p-2 \geq 0$ and $a_{v} \downarrow 0$, we get

$$
\begin{aligned}
I_{2} & =\sum_{\mu=1}^{n} a_{\mu}^{p} \mu^{(k+1) p-2} \\
& \leq \sum_{\mu=1}^{[n / 2]} a_{\mu}^{p} \mu^{(k+1) p-2}+a_{[n / 2]+1}^{p} \sum_{\mu=[n / 2]+1}^{n} \mu^{(k+1) p-2} \\
& \leq \sum_{\mu=1}^{[n / 2]} a_{\mu}^{p} \mu^{(k+1) p-2}+C_{8} n^{(k+1) p-1} a_{[n / 2]+1}^{p} \\
& \leq C_{9} \sum_{\mu=1}^{[n / 2]} a_{\mu}^{p} \mu^{(k+1) p-2} .
\end{aligned}
$$

Since $k-r-\lambda>0$, we have

$$
\begin{aligned}
I_{1}^{\theta / p} & \leq C_{10} n^{-(k-r) \theta}\left(\sum_{\mu=1}^{[n / 2]} a_{\mu}^{p} \mu^{(k+1) p-2}\right)^{\theta / p} \\
& \leq C_{11} n^{-\lambda \theta} \sum_{\nu=[n / 2]}^{n} \nu^{-(k-r-\lambda) \theta-1}\left(\sum_{\mu=1}^{\nu} a_{\mu}^{p} \mu^{(k+1) p-2}\right)^{\theta / p} \\
& \leq C_{11} n^{-\lambda \theta} \sum_{\nu=1}^{n} \nu^{-(k-r-\lambda) \theta-1}\left(\sum_{\mu=1}^{\nu} a_{\mu}^{p} \mu^{(k+1) p-2}\right)^{\theta / p}
\end{aligned}
$$

Applying Lemma 12, we obtain

$$
\begin{aligned}
I_{1}^{\theta / p} & \leq C_{12} n^{-\lambda \theta} \sum_{\nu=1}^{n} \nu^{-(k-r-\lambda) \theta-1}\left(a_{\nu}^{p} \nu^{(k+1) p-2} \nu\right)^{\theta / p} \\
& =C_{12} n^{-\lambda \theta} \sum_{\nu=1}^{n} a_{\nu}^{\theta} \nu^{(r+\lambda) \theta+\theta-\theta / p-1} .
\end{aligned}
$$

From (50), it follows that

$$
\begin{aligned}
J_{1} \leq C_{13}\left(\sum_{\nu=[(n+1) / 4]}^{\infty} a_{\nu}^{\theta} \nu^{r \theta+\theta-\theta / p-1}\right. \\
\left.\quad+n^{-\lambda \theta} \sum_{\nu=1}^{n} a_{\nu}^{\theta} \nu^{(r+\lambda) \theta+\theta-\theta / p-1}\right) .
\end{aligned}
$$

This way, inequalities (46), (47), (48), and (56) yield

$$
\begin{aligned}
C_{14} & \sum_{\nu=8(n+1)}^{\infty} a_{\nu}^{\theta} \nu^{r \theta+\theta-\theta / p-1} \leq J_{1}+J_{2} \\
\leq & C_{15}\left(\sum_{\nu=[(n+1) / 4]}^{\infty} a_{\nu}^{\theta} \nu^{r \theta+\theta-\theta / p-1}\right. \\
& \left.+n^{-\lambda \theta} \sum_{\nu=1}^{n} a_{\nu}^{\theta} \nu^{(r+\lambda) \theta+\theta-\theta / p-1}\right) .
\end{aligned}
$$

Now we estimate $J_{3}$ and $J_{4}$. Put

$$
\begin{aligned}
& A_{1}=n^{\lambda \theta} J_{3}=\sum_{\nu=1}^{n} \nu^{(r+\lambda-k) \theta-1}\left(\sum_{\mu=1}^{\nu} a_{\mu}^{p} \mu^{(k+1) p-2}\right)^{\theta / p}, \\
& A_{2}=n^{\lambda \theta} J_{4}=\sum_{\nu=1}^{n} \nu^{(r+\lambda) \theta-1}\left(\sum_{\mu=\nu}^{\infty} a_{\mu}^{p} \mu^{p-2}\right)^{\theta / p} .
\end{aligned}
$$

Applying Lemma 12, for $r+\lambda-k<0$, we get

$$
A_{1} \leq C_{16} \sum_{\nu=1}^{n} a_{\nu}^{\theta} \nu^{(r+\lambda) \theta+\theta-\theta / p-1}
$$

We estimate $A_{2}$ in an analogous way:

$$
\begin{aligned}
A_{2} & \leq C_{17}\left(\sum_{\nu=1}^{n} \nu^{(r+\lambda) \theta-1}\left(\sum_{\mu=\nu}^{n} a_{\mu}^{p} \mu^{p-2}\right)^{\theta / p}\right. \\
& \left.+\sum_{\nu=1}^{n} \nu^{(r+\lambda) \theta-1}\left(\sum_{\mu=n+1}^{\infty} a_{\mu}^{p} \mu^{p-2}\right)^{\theta / p}\right) \\
& \leq C_{18}\left(\sum_{\nu=1}^{n} a_{\nu}^{\theta} \nu^{(r+\lambda) \theta+\theta-\theta / p-1}\right. \\
& \left.+n^{(r+\lambda) \theta}\left(\sum_{\mu=n+1}^{\infty} a_{\mu}^{p} \mu^{p-2}\right)^{\theta / p}\right) .
\end{aligned}
$$

We estimate the series

$$
B=\left(\sum_{\mu=n+1}^{\infty} a_{\mu}^{p} \mu^{p-2}\right)^{\theta / p}
$$


First let $\theta / p>1$. Applying Hölder's inequality, we have

$$
\begin{aligned}
\sum_{\mu=n+1}^{\infty} a_{\mu}^{p} \mu^{p-2} \leq & \left(\sum_{\mu=n+1}^{\infty}\left(a_{\mu}^{p} \mu^{p-1+r p-p / \theta}\right)^{\theta / p}\right)^{p / \theta} \\
& \times\left(\sum_{\mu=n+1}^{\infty} \mu^{-(r p-p / \theta+1) \theta /(\theta-p)}\right)^{(\theta-p) / \theta} .
\end{aligned}
$$

Since $(r p-p / \theta+1)(\theta /(\theta-p))=r p(\theta /(\theta-p))+1>1$, we get

$$
\sum_{\mu=n+1}^{\infty} a_{\mu}^{p} \mu^{p-2} \leq C_{19} n^{-r p}\left(\sum_{\mu=n+1}^{\infty} a_{\mu}^{\theta} \mu^{\theta-\theta / p+r \theta-1}\right)^{p / \theta} .
$$

So, for $\theta / p>1$, we have proven that

$$
B \leq C_{20} n^{-r \theta} \sum_{\mu=n+1}^{\infty} a_{\mu}^{\theta} \mu^{r \theta+\theta-\theta / p-1} .
$$

Let $\theta / p \leq 1$. For given $n$, we choose the positive integer $N$ such that $2^{N} \leq n+1<2^{N+1}$. Then, we have

$$
\begin{aligned}
B & \leq\left(\sum_{\mu=2^{N}}^{\infty} a_{\mu}^{p} \mu^{p-2}\right)^{\theta / p} \leq\left(\sum_{\nu=N}^{\infty} a_{2^{\nu}}^{p} \sum_{\mu=2^{\nu}}^{2^{\nu+1}-1} \mu^{p-2}\right)^{\theta / p} \\
& \leq C_{21}\left(\sum_{\nu=N}^{\infty} a_{2^{\nu}}^{p} 2^{\nu(p-1)}\right)^{\theta / p} .
\end{aligned}
$$

Making use of Lemma 9, we obtain

$$
\begin{aligned}
B & \leq C_{21} \sum_{\nu=N}^{\infty} a_{2^{\nu}}^{\theta} 2^{\nu(\theta-\theta / p)} \leq C_{22} \sum_{\nu=N}^{\infty} \sum_{\mu=2^{\nu-1}}^{2^{\nu}-1} a_{\mu}^{\theta} \mu^{\theta-\theta / p-1} \\
& =C_{22} \sum_{\nu=2^{N-1}}^{\infty} a_{\nu}^{\theta} \nu^{\theta-\theta / p-1} \leq C_{22} \sum_{\nu=[(n+1) / 4]}^{\infty} a_{\nu}^{\theta} \nu^{\theta-\theta / p-1} \\
& \leq C_{22}\left[\frac{n+1}{4}\right]^{-r \theta} \sum_{\nu=[(n+1) / 4]}^{\infty} a_{\nu}^{\theta} \nu^{r \theta+\theta-\theta / p-1} .
\end{aligned}
$$

Since, for $n \geq 3,[(n+1) / 4] \geq n / 12$ holds, we get

$$
B \leq C_{23} n^{-r \theta} \sum_{\nu=[(n+1) / 4]}^{\infty} a_{\nu}^{\theta} \nu^{r \theta+\theta-\theta / p-1} .
$$

This way, for $0<\theta / p<\infty$, we proved that

$$
B \leq C_{24} n^{-r \theta} \sum_{\nu=[(n+1) / 4]}^{\infty} a_{\nu}^{\theta} \nu^{r \theta+\theta-\theta / p-1} .
$$

Hence, (60) yields

$$
\begin{aligned}
A_{2} \leq C_{25}\left(\sum_{\nu=1}^{n} a_{\nu}^{\theta} \nu^{(r+\lambda) \theta+\theta-\theta / p-1}\right. \\
\left.+n^{\lambda \theta} \sum_{\nu=[(n+1) / 4]}^{\infty} a_{\nu}^{\theta} \nu^{r \theta+\theta-\theta / p-1}\right) .
\end{aligned}
$$

Now, from (59), it follows that

$$
\begin{aligned}
J_{3} & +J_{4}=n^{-\lambda \theta}\left(A_{1}+A_{2}\right) \\
& \leq C_{26}\left(n^{-\lambda \theta} \sum_{\nu=1}^{n} a_{\nu}^{\theta} \nu^{(r+\lambda) \theta+\theta-\theta / p-1}\right. \\
& \left.+\sum_{\nu=[(n+1) / 4]}^{\infty} a_{\nu}^{\theta} v^{r \theta+\theta-\theta / p-1}\right) .
\end{aligned}
$$

Further, we estimate the series

$$
\begin{aligned}
A_{3} & =\sum_{\nu=[(n+1) / 4]}^{\infty} a_{\nu}^{\theta} \nu^{r \theta+\theta-\theta / p-1} \\
& =A_{4}+\sum_{\nu=n+1}^{\infty} a_{\nu}^{\theta} \nu^{r \theta+\theta-\theta / p-1},
\end{aligned}
$$

where

$$
\begin{aligned}
A_{4} & =\sum_{\nu=[(n+1) / 4]}^{n} a_{\nu}^{\theta} \nu^{r \theta+\theta-\theta / p-1} \leq C_{27} a_{[(n+1) / 4]}^{\theta} n^{r \theta+\theta-\theta / p} \\
& \leq C_{28} n^{-\lambda \theta} \sum_{\nu=1}^{[(n+1) / 4]} a_{\nu}^{\theta} \nu^{(r+\lambda) \theta+\theta-\theta / p-1} \\
& \leq C_{28} n^{-\lambda \theta} \sum_{\nu=1}^{n} a_{\nu}^{\theta} \nu^{(r+\lambda) \theta+\theta-\theta / p-1}
\end{aligned}
$$

Hence

$$
\begin{aligned}
A_{3} & \leq C_{29}\left(n^{-\lambda \theta} \sum_{\nu=1}^{n} a_{\nu}^{\theta} \nu^{(r+\lambda) \theta+\theta-\theta / p-1}\right. \\
& \left.+\sum_{\nu=n+1}^{\infty} a_{\nu}^{\theta} \nu^{r \theta+\theta-\theta / p-1}\right)
\end{aligned}
$$

Making use of (73) and (70), we have

$$
\begin{aligned}
J_{3} & +J_{4} \leq C_{30}\left(n^{-\lambda \theta} \sum_{\nu=1}^{n} a_{\nu}^{\theta} \nu^{(r+\lambda) \theta+\theta-\theta / p-1}\right. \\
& \left.+\sum_{\nu=n+1}^{\infty} a_{\nu}^{\theta} \nu^{r \theta+\theta-\theta / p-1}\right)
\end{aligned}
$$

Hence, applying (73) in (57), we obtain

$$
\begin{aligned}
J_{1} & +J_{2}+J_{3}+J_{4} \leq C_{31}\left(n^{-\lambda \theta} \sum_{\nu=1}^{n} a_{\nu}^{\theta} \nu^{(r+\lambda) \theta+\theta-\theta / p-1}\right. \\
& \left.+\sum_{\nu=n+1}^{\infty} a_{\nu}^{\theta} \nu^{r \theta+\theta-\theta / p-1}\right) .
\end{aligned}
$$

Now we estimate $A_{1}$ and $A_{2}$ from below. Making use of Lemma 12, we get

$$
A_{1} \geq C_{32} \sum_{\nu=1}^{n} a_{\nu}^{\theta} \nu^{(r+\lambda) \theta+\theta-\theta / p-1}
$$


and, in an analogous way,

$$
\begin{aligned}
A_{2} & \geq \sum_{\nu=1}^{n} \nu^{(r+\lambda) \theta-1}\left(\sum_{\mu=v}^{n} a_{\mu}^{p} \mu^{p-2}\right)^{\theta / p} \\
& \geq C_{33} \sum_{\nu=1}^{n} a_{\nu}^{\theta} \nu^{(r+\lambda) \theta+\theta-\theta / p-1} .
\end{aligned}
$$

Hence,

$$
A_{1}+A_{2} \geq C_{34} \sum_{\nu=1}^{n} a_{\nu}^{\theta} \nu^{(r+\lambda) \theta+\theta-\theta / p-1} .
$$

This way the following inequality holds:

$$
J_{3}+J_{4} \geq C_{35} n^{-\lambda \theta} \sum_{\nu=1}^{n} a_{\nu}^{\theta} \nu^{(r+\lambda) \theta+\theta-\theta / p-1} .
$$

From (57), it follows that

$$
\begin{aligned}
J_{1} & +J_{2}+J_{3}+J_{4} \geq C_{36}\left(\sum_{\nu=8(n+1)}^{\infty} a_{\nu}^{\theta} \nu^{r \theta+\theta-\theta / p-1}\right. \\
& \left.+n^{-\lambda \theta} \sum_{\nu=1}^{n} a_{\nu}^{\theta} \nu^{(r+\lambda) \theta+\theta-\theta / p-1}\right) .
\end{aligned}
$$

Since

$$
\begin{aligned}
& \sum_{\nu=n+1}^{\nu=8(n+1)-1} a_{\nu}^{\theta} \nu^{r \theta+\theta-\theta / p-1} \leq C_{37} a_{n}^{\theta} n^{r \theta+\theta-\theta / p} \\
& \leq C_{38} n^{-\lambda \theta} \sum_{\nu=1}^{n} a_{\nu}^{\theta} \nu^{(r+\lambda) \theta+\theta-\theta / p-1}
\end{aligned}
$$

holds, we have

$$
\begin{aligned}
& \sum_{\nu=n+1}^{\infty} a_{\nu}^{\theta} \nu^{r \theta+\theta-\theta / p-1}+n^{-\lambda \theta} \sum_{\nu=1}^{n} a_{\nu}^{\theta} \nu^{(r+\lambda) \theta+\theta-\theta / p-1} \\
& \leq C_{39}\left(\sum_{\nu=8(n+1)}^{\infty} a_{\nu}^{\theta} \nu^{r \theta+\theta-\theta / p-1}\right. \\
& \left.+n^{-\lambda \theta} \sum_{\nu=1}^{n} a_{\nu}^{\theta} \nu^{(r+\lambda) \theta+\theta-\theta / p-1}\right) .
\end{aligned}
$$

Now, estimates (80) and (75) imply that

$$
\begin{aligned}
& C_{40}\left(\sum_{\nu=n+1}^{\infty} a_{\nu}^{\theta} \nu^{r \theta+\theta-\theta / p-1}+n^{-\lambda \theta} \sum_{\nu=1}^{n} a_{\nu}^{\theta} \nu^{(r+\lambda) \theta+\theta-\theta / p-1}\right) \\
& \leq J_{1}+J_{2}+J_{3}+J_{4} \leq C_{41}\left(\sum_{\nu=n+1}^{\infty} a_{\nu}^{\theta} \nu^{r \theta+\theta-\theta / p-1}\right. \\
& \left.+n^{-\lambda \theta} \sum_{\nu=1}^{n} a_{\nu}^{\theta} \nu^{(r+\lambda) \theta+\theta-\theta / p-1}\right) .
\end{aligned}
$$

This way we proved that condition (9) is equivalent to the condition of the theorem. Since condition (9) is equivalent to the condition $f \in N(p, \theta, r, \lambda, \varphi)$, proof of Theorem 2 is completed.
Proof of Theorem 5. Considering Lemma 14, condition $f \in$ $N(p, \theta, r, \lambda, \varphi)$ is equivalent to the condition

$$
\begin{aligned}
& \sum_{\nu=n+1}^{\infty} 2^{\nu r \theta} E_{2^{\nu}}(f)_{p}^{\theta}+2^{-n \lambda \theta} \sum_{\nu=0}^{n} 2^{\nu(r+\lambda) \theta} E_{2^{\nu}}(f)_{p}^{\theta} \\
& \quad \leq C_{42} \varphi\left(\frac{1}{2^{n}}\right)^{\theta},
\end{aligned}
$$

where constant $C$ does not depend on $n$. Corollary 16 yields that the last estimate is equivalent to the estimate

$$
\begin{aligned}
& \sum_{\nu=n+1}^{\infty} 2^{\nu r \theta}\left(\sum_{\mu=\nu}^{\infty} a_{\mu}^{2}\right)^{\theta / 2}+2^{-n \lambda \theta} \sum_{\nu=0}^{n} 2^{\nu(r+\lambda) \theta}\left(\sum_{\mu=\nu}^{\infty} a_{\mu}^{2}\right)^{\theta / 2} \\
& \leq C_{43} \varphi\left(\frac{1}{2^{n}}\right)^{\theta},
\end{aligned}
$$

where constant $C_{43}$ does not depend on $n$.

Put

$$
\begin{aligned}
& J_{1}=\sum_{\nu=n+1}^{\infty} 2^{\nu r \theta}\left(\sum_{\mu=\nu}^{\infty} a_{\mu}^{2}\right)^{\theta / 2}, \\
& J_{2}=2^{-n \lambda \theta} \sum_{\nu=0}^{n} 2^{\nu(r+\lambda) \theta}\left(\sum_{\mu=\nu}^{\infty} a_{\mu}^{2}\right)^{\theta / 2} ;
\end{aligned}
$$

we estimate $J_{1}$ and $J_{2}$ from below and above.

Let $0<\theta / 2 \leq 1$. Using Lemma 9 , changing the order of summation, we get

$$
J_{1} \leq \sum_{\nu=n+1}^{\infty} 2^{\nu r \theta} \sum_{\mu=v}^{\infty} a_{\mu}^{\theta}=\sum_{\mu=n+1}^{\infty} a_{\mu}^{\theta} \sum_{\nu=n+1}^{\mu} 2^{\nu r \theta} .
$$

Therefrom, taking into consideration the fact that $r \theta>0$ while computing the second sum, we obtain

$$
J_{1} \leq C_{44} \sum_{\mu=n+1}^{\infty} a_{\mu}^{\theta} 2^{\mu r \theta} .
$$

Let $1 \leq \theta / 2<\infty$ and $0<\varepsilon<r$. Applying Hölder's inequality, we have

$$
A=\sum_{\mu=\nu}^{\infty} a_{\mu}^{2} \leq\left(\sum_{\mu=\nu}^{\infty} a_{\mu}^{\theta} 2^{\mu \varepsilon \theta}\right)^{2 / \theta}\left(\sum_{\mu=\nu}^{\infty} 2^{-2 \mu \varepsilon \theta^{\prime}}\right)^{1 / \theta^{\prime}},
$$

where $2 / \theta+1 / \theta^{\prime}=1$. Computing the second sum, we obtain

$$
A \leq \frac{C_{45}}{2^{2 \varepsilon v}}\left(\sum_{\mu=\nu}^{\infty} a_{\mu}^{\theta} 2^{\mu \varepsilon \theta}\right)^{2 / \theta} .
$$

Now we have

$$
\begin{aligned}
J_{1} & \leq C_{46} \sum_{\nu=n+1}^{\infty} 2^{\nu(r-\varepsilon) \theta} \sum_{\mu=v}^{\infty} a_{\mu}^{\theta} 2^{\mu \varepsilon \theta} \\
& =C_{46} \sum_{\mu=n+1}^{\infty} a_{\mu}^{\theta} 2^{\mu \varepsilon \theta} \sum_{\nu=n+1}^{\mu} 2^{\nu(r-\varepsilon) \theta} \leq C_{47} \sum_{\mu=n+1}^{\infty} a_{\mu}^{\theta} 2^{\mu r \theta} .
\end{aligned}
$$


This way, for $0<\theta<\infty$, we have

$$
J_{1} \leq C_{48} \sum_{\mu=n+1}^{\infty} a_{\mu}^{\theta} 2^{\mu r \theta},
$$

where constant $C_{48}$ does not depend on $n$.

Now we estimate $J_{1}$ from below.

Let $1 \leq \theta / 2<\infty$. Making use of Lemma 9, we get

$$
J_{1} \geq \sum_{\nu=n+1}^{\infty} 2^{\nu r \theta} \sum_{\mu=v}^{\infty} a_{\mu}^{\theta}=\sum_{\mu=n+1}^{\infty} a_{\mu}^{\theta} \sum_{\nu=n+1}^{\mu} 2^{\nu r \theta} .
$$

Computing the second sum, we get

$$
J_{1} \geq C_{49} \sum_{\mu=n+1}^{\infty} a_{\mu}^{\theta} 2^{\mu r \theta} .
$$

Let $0<\theta / 2 \leq 1$ and $\varepsilon>0$. Applying Hölder's inequality, we have

$$
\begin{aligned}
\sum_{\mu=\nu}^{\infty} a_{\mu}^{\theta} 2^{-\mu \varepsilon \theta} & \leq\left(\sum_{\mu=\nu}^{\infty} a_{\mu}^{2}\right)^{\theta / 2}\left(\sum_{\mu=\nu}^{\infty} 2^{-\mu \varepsilon \theta \theta^{\prime}}\right)^{1 / \theta^{\prime}} \\
& \leq \frac{C_{50}}{2^{\nu \varepsilon \theta}}\left(\sum_{\mu=\nu}^{\infty} a_{\mu}^{2}\right)^{\theta / 2}
\end{aligned}
$$

where $\theta / 2+1 / \theta^{\prime}=1$. The last estimate implies that

$$
J_{1} \geq C_{51} \sum_{\nu=n+1}^{\infty} 2^{\nu(r+\varepsilon) \theta} \sum_{\mu=\nu}^{\infty} a_{\mu}^{\theta} 2^{-\mu \varepsilon \theta} .
$$

Changing the order of summation and then computing the second sum, we obtain

$$
J_{1} \geq C_{51} \sum_{\mu=n+1}^{\infty} a_{\mu}^{\theta} 2^{-\mu \varepsilon \theta} \sum_{\nu=n+1}^{\mu} 2^{\nu(r+\varepsilon) \theta} \geq C_{52} \sum_{\mu=n+1}^{\infty} a_{\mu}^{\theta} 2^{\mu r \theta},
$$

where constant $C_{52}$ does not depend on $n$.

Consequently, for every $0<\theta<\infty$, the following estimate holds:

$$
C_{53} \sum_{\mu=n+1}^{\infty} a_{\mu}^{\theta} 2^{\mu r \theta} \leq J_{1} \leq C_{54} \sum_{\mu=n+1}^{\infty} a_{\mu}^{\theta} 2^{\mu r \theta}
$$

where constants $C_{53}$ and $C_{54}$ do not depend on $n$.

Now we estimate $J_{2}$. Obviously,

$$
J_{2} \geq 2^{-n \lambda \theta} \sum_{\nu=0}^{n} 2^{\nu(r+\lambda) \theta}\left(\sum_{\mu=\nu}^{n} a_{\mu}^{2}\right)^{\theta / 2} .
$$

Let $1 \leq \theta / 2<\infty$. Applying Lemma 9, changing the order of summation and then computing the second sum, we obtain

$$
\begin{aligned}
J_{2} & \geq 2^{-n \lambda \theta} \sum_{\nu=0}^{n} 2^{\nu(r+\lambda) \theta} \sum_{\mu=\nu}^{n} a_{\mu}^{\theta}=2^{-n \lambda \theta} \sum_{\mu=0}^{n} a_{\mu}^{\theta} \sum_{\nu=0}^{\mu} 2^{\nu(r+\lambda) \theta} \\
& \geq C_{55} 2^{-n \lambda \theta} \sum_{\mu=0}^{n} a_{\mu}^{\theta} 2^{\mu(r+\lambda) \theta} .
\end{aligned}
$$

Let $0<\theta / 2 \leq 1$ and $\varepsilon>0$. Applying Hölder's inequality, we get

$$
\begin{aligned}
\sum_{\mu=\nu}^{n} a_{\mu}^{\theta} 2^{-\mu \varepsilon \theta} & \leq\left(\sum_{\mu=\nu}^{n} a_{\mu}^{2}\right)^{\theta / 2}\left(\sum_{\mu=\nu}^{n} 2^{-\mu \varepsilon \theta \theta^{\prime}}\right)^{1 / \theta^{\prime}} \\
& \leq \frac{C_{56}}{2^{\nu \varepsilon \theta}}\left(\sum_{\mu=\nu}^{n} a_{\mu}^{2}\right)^{\theta / 2}
\end{aligned}
$$

where $\theta / 2+1 / \theta^{\prime}=1$. The last estimate implies that

$$
J_{2} \geq C_{57} 2^{-n \lambda \theta} \sum_{\nu=0}^{n} 2^{\nu(r+\lambda+\varepsilon) \theta} \sum_{\mu=\nu}^{n} a_{\mu}^{\theta} 2^{-\mu \varepsilon \theta}
$$

Changing the order of summation and computing the second sum, we have

$$
\begin{aligned}
J_{2} & \geq C_{57} 2^{-n \lambda \theta} \sum_{\mu=0}^{n} a_{\mu}^{\theta} 2^{-\mu \varepsilon \theta} \sum_{\nu=0}^{\mu} 2^{\nu(r+\lambda+\varepsilon) \theta} \\
& \geq C_{58} 2^{-n \lambda \theta} \sum_{\mu=0}^{n} a_{\mu}^{\theta} 2^{\mu(r+\lambda) \theta} .
\end{aligned}
$$

Thus, for every $0<\theta<\infty$, the following holds:

$$
J_{2} \geq C_{59} 2^{-n \lambda \theta} \sum_{\mu=0}^{n} a_{\mu}^{\theta} 2^{\mu(r+\lambda) \theta} .
$$

Now we estimate $J_{2}$ from above. Taking into consideration the fact that $(r+\lambda) \theta>0$, we have

$$
\begin{aligned}
J_{2} & \leq C_{60} 2^{-n \lambda \theta} \sum_{\nu=0}^{n} 2^{\nu(r+\lambda) \theta}\left(\left(\sum_{\mu=\nu}^{n} a_{\mu}^{2}\right)^{\theta / 2}\right. \\
& \left.+\left(\sum_{\mu=n+1}^{\infty} a_{\mu}^{2}\right)^{\theta / 2}\right) \\
& \leq C_{61}\left(2^{-n \lambda \theta} \sum_{\nu=0}^{n} 2^{\nu(r+\lambda) \theta}\left(\sum_{\mu=\nu}^{n} a_{\mu}^{2}\right)^{\theta / 2}\right. \\
& \left.+2^{n r \theta}\left(\sum_{\mu=n+1}^{\infty} a_{\mu}^{2}\right)^{\theta / 2}\right) .
\end{aligned}
$$

Since

$$
2^{n r \theta}\left(\sum_{\mu=n+1}^{\infty} a_{\mu}^{2}\right)^{\theta / 2} \leq \sum_{\mu=n+1}^{\infty} 2^{v r \theta}\left(\sum_{\mu=n+1}^{\infty} a_{\mu}^{2}\right)^{\theta / 2}=J_{1}
$$


holds and an upper bound for $J_{1}$ is already found, we estimate from above the expression

$$
J_{3}=\sum_{\nu=0}^{n} 2^{\nu(r+\lambda) \theta}\left(\sum_{\mu=\nu}^{n} a_{\mu}^{2}\right)^{\theta / 2}
$$

Let $0<\theta / 2 \leq 1$. Applying Lemma 9, we obtain

$$
\begin{aligned}
J_{3} & \leq \sum_{\nu=0}^{n} 2^{\nu(r+\lambda) \theta} \sum_{\mu=\nu}^{n} a_{\mu}^{\theta}=\sum_{\mu=0}^{n} a_{\mu}^{\theta} \sum_{\nu=0}^{\mu} 2^{\nu(r+\lambda) \theta} \\
& \leq C_{62} \sum_{\mu=0}^{n} a_{\mu}^{\theta} 2^{\mu(r+\lambda) \theta} .
\end{aligned}
$$

Let $1 \leq \theta / 2<\infty$ and $0<\varepsilon<r+\lambda$. Then, applying Hölder's inequality, we have

$$
\sum_{\mu=\nu}^{n} a_{\mu}^{2} \leq\left(\sum_{\mu=\nu}^{n} a_{\mu}^{\theta} 2^{\mu \varepsilon \theta}\right)^{2 / \theta}\left(\sum_{\mu=\nu}^{n} 2^{-2 \mu \varepsilon \theta^{\prime}}\right)^{1 / \theta^{\prime}}
$$

where $2 / \theta+1 / \theta^{\prime}=1$. Using the last estimate, we get

$$
\begin{aligned}
J_{3} & \leq \sum_{\nu=0}^{n} 2^{\nu(r+\lambda) \theta}\left(\sum_{\mu=\nu}^{\infty} 2^{-2 \mu \varepsilon \theta^{\prime}}\right)^{\theta / 2 \theta^{\prime}} \sum_{\mu=\nu}^{n} a_{\mu}^{\theta} 2^{\mu \varepsilon \theta} \\
& \leq C_{63} \sum_{\nu=0}^{n} 2^{\nu(r+\lambda-\varepsilon) \theta} \sum_{\mu=\nu}^{n} a_{\mu}^{\theta} 2^{\mu \varepsilon \theta} .
\end{aligned}
$$

Changing the order of summation and computing the second sum, we obtain

$$
J_{3} \leq C_{63} \sum_{\mu=0}^{n} a_{\mu}^{\theta} 2^{\mu \varepsilon \theta} \sum_{\nu=0}^{\mu} 2^{\nu(r+\lambda-\varepsilon) \theta} \leq C_{64} \sum_{\mu=0}^{n} a_{\mu}^{\theta} 2^{\mu(r+\lambda) \theta}
$$

Therefore, for every $0<\theta<\infty$, the following estimate holds:

$$
J_{3} \leq C_{65} \sum_{\mu=0}^{n} a_{\mu}^{\theta} 2^{\mu(r+\lambda) \theta}
$$

Now, making use of inequalities (105) and (98), we have

$$
J_{2} \leq C_{66}\left(2^{-n \lambda \theta} \sum_{\mu=0}^{n} a_{\mu}^{\theta} 2^{\mu(r+\lambda) \theta}+\sum_{\mu=n+1}^{\infty} a_{\mu}^{\theta} 2^{\mu r \theta}\right) .
$$

This way, inequalities (98) and (104) and the last inequality imply the estimate

$$
\begin{gathered}
C_{67}\left(\sum_{\mu=n+1}^{\infty} a_{\mu}^{\theta} 2^{\mu r \theta}+2^{-n \lambda \theta} \sum_{\mu=0}^{n} a_{\mu}^{\theta} 2^{\mu(r+\lambda) \theta}\right) \leq J_{1}+J_{2} \\
\quad \leq C_{68}\left(\sum_{\mu=n+1}^{\infty} a_{\mu}^{\theta} 2^{\mu r \theta}+2^{-n \lambda \theta} \sum_{\mu=0}^{n} a_{\mu}^{\theta} 2^{\mu(r+\lambda) \theta}\right),
\end{gathered}
$$

where constants $C_{67}$ and $C_{68}$ do not depend on $n$. Hence, considering condition (85), we conclude that condition $f \in$ $N(p, \theta, r, \lambda, \varphi)$ is equivalent to the condition

$$
\begin{aligned}
A_{n} & =\sum_{\mu=n+1}^{\infty} a_{\mu}^{\theta} 2^{\mu r \theta}+2^{-n \lambda \theta} \sum_{\mu=0}^{n} a_{\mu}^{\theta} 2^{\mu(r+\lambda) \theta} \\
& \leq C_{69} \varphi\left(\frac{1}{2^{n}}\right)^{\theta},
\end{aligned}
$$

where constant $C_{69}$ does not depend on $n$.

We put

$$
D_{m}=\sum_{\nu=m+1}^{\infty} \lambda_{\nu}^{\theta} \nu^{r \theta}+m^{-\lambda \theta} \sum_{\nu=1}^{m} \lambda_{\nu}^{\theta} \nu^{(r+\lambda) \theta} .
$$

For given $m$, we choose the positive integer $n$ such that $2^{n} \leq$ $m+1<2^{n+1}$.

First we consider the case $2^{n}<m+1<2^{n+1}$. We have

$$
\begin{aligned}
D_{m}= & \sum_{\nu=2^{n+1}}^{\infty} \lambda_{\nu}^{\theta} \nu^{r \theta}+\sum_{\nu=m+1}^{2^{n+1}-1} \lambda_{\nu}^{\theta} \nu^{r \theta}+m^{-\lambda \theta} \sum_{\nu=1}^{2^{n}-1} \lambda_{\nu}^{\theta} \nu^{(r+\lambda) \theta} \\
& +m^{-\lambda \theta} \sum_{\nu=2^{n}}^{m} \lambda_{\nu}^{\theta} \nu^{(r+\lambda) \theta} .
\end{aligned}
$$

Since $\lambda_{\nu}=0$ for $\nu \neq 2^{\mu}$, we get

$$
\begin{aligned}
D_{m}= & \sum_{\nu=2^{n+1}}^{\infty} \lambda_{\nu}^{\theta} \nu^{r \theta}+m^{-\lambda \theta} \sum_{\nu=1}^{2^{n}-1} \lambda_{\nu}^{\theta} \nu^{(r+\lambda) \theta} \\
& +m^{-\lambda \theta} \lambda_{2^{n}}^{\theta} 2^{n(r+\lambda) \theta} \\
= & \sum_{\mu=n+1}^{\infty} \sum_{\nu=2^{\mu}}^{2^{\mu+1}-1} \lambda_{\nu}^{\theta} \nu^{r \theta}+m^{-\lambda \theta} \sum_{\mu=0}^{n-1} \sum_{\nu=2^{\mu}}^{2^{\mu+1}-1} \lambda_{\nu}^{\theta} \nu^{(r+\lambda) \theta} \\
& +m^{-\lambda \theta} \lambda_{2^{n}}^{\theta} 2^{n(r+\lambda) \theta} \\
= & \sum_{\mu=n+1}^{\infty} \lambda_{2^{\mu}}^{\theta} 2^{\mu r \theta}+m^{-\lambda \theta} \sum_{\mu=0}^{n-1} \lambda_{2^{\mu}}^{\theta} 2^{\mu(r+\lambda) \theta} \\
& +m^{-\lambda \theta} \lambda_{2^{n}}^{\theta} 2^{n(r+\lambda) \theta} .
\end{aligned}
$$

Further, since $\lambda_{2^{\mu}}=a_{\mu}$, we get

$$
D_{m}=\sum_{\mu=n+1}^{\infty} a_{\mu}^{\theta} 2^{\mu r \theta}+m^{-\lambda \theta} \sum_{\mu=0}^{n} a_{\mu}^{\theta} 2^{\mu(r+\lambda) \theta} .
$$

Hence, for $2^{n}<m+1<2^{n+1}$, we obtain

$$
\begin{gathered}
C_{70}\left(\sum_{\mu=n+1}^{\infty} a_{\mu}^{\theta} 2^{\mu r \theta}+2^{-n \lambda \theta} \sum_{\mu=0}^{n} a_{\mu}^{\theta} 2^{\mu(r+\lambda) \theta}\right) \leq D_{m} \\
\leq C_{71}\left(\sum_{\mu=n+1}^{\infty} a_{\mu}^{\theta} 2^{\mu r \theta}+2^{-n \lambda \theta} \sum_{\mu=0}^{n} a_{\mu}^{\theta} 2^{\mu(r+\lambda) \theta}\right),
\end{gathered}
$$

where constants $C_{70}$ and $C_{71}$ do not depend on $m$ and $n$. 
Let us assume now that $m+1=2^{n}$. In an analogous way, we have

$$
\begin{aligned}
D_{m} & =\sum_{\nu=2^{n}}^{\infty} \lambda_{\nu}^{\theta} \nu^{r \theta}+2^{-n \lambda \theta^{2^{n}-1}} \sum_{\nu=1}^{\theta} \lambda_{\nu}^{\theta} \nu^{(r+\lambda) \theta} \\
& =\sum_{\mu=n}^{\infty} a_{\mu}^{\theta} 2^{\mu r \theta}+2^{-n \lambda \theta} \sum_{\mu=0}^{n-1} a_{\mu}^{\theta} 2^{\mu(r+\lambda) \theta} \\
& =\sum_{\mu=n+1}^{\infty} a_{\mu}^{\theta} 2^{\mu r \theta}+2^{-n \lambda \theta} \sum_{\mu=0}^{n} a_{\mu}^{\theta} 2^{\mu(r+\lambda) \theta}=A_{n} .
\end{aligned}
$$

Thus, for $2^{n} \leq m+1<2^{n+1}$, the following estimate holds:

$$
C_{72} A_{n} \leq D_{m} \leq C_{73} A_{n}
$$

where constants $C_{72}$ and $C_{73}$ do not depend on $m$ and $n$. Hence, considering condition (115), we conclude that condition $f \in N(p, \theta, r, \lambda, \varphi)$ is equivalent to the condition

$$
D_{m} \leq C_{74} \varphi\left(\frac{1}{2^{n}}\right)^{\theta}
$$

where constant $C_{74}$ does not depend on $m$ and $n$.

Since $1 / 2^{n}<2 /(m+1)<2 / m$, we get

$$
\varphi\left(\frac{1}{2^{n}}\right) \leq C_{75} \varphi\left(\frac{2}{m+1}\right) \leq C_{76} \varphi\left(\frac{2}{m}\right)
$$

where constant $C_{76}$ does not depend on $m$ and $n$; and since $1 / 2^{n} \geq 1 /(m+1) \geq 1 / 2 m$, we get

$$
\varphi\left(\frac{1}{2^{n}}\right) \geq C_{77} \varphi\left(\frac{1}{2 m}\right) \geq C_{78} \varphi\left(\frac{1}{m}\right),
$$

where constant $C_{78}$ does not depend on $m$ and $n$. This way, condition (123) is equivalent to the condition

$$
D_{m} \leq C_{79} \varphi\left(\frac{1}{m}\right)^{\theta},
$$

where constant $C_{79}$ does not depend on $m$.

This completes the proof of Theorem 5 .

Remark 17. Notice that another way of proving Theorems 2 and 5 is presented in [2]. Our approach here is similar to that used in [16].

\section{Conflicts of Interest}

The authors declare that there are no conflicts of interest regarding the publication of this paper.

\section{References}

[1] B. Laković, “Ob odnom klasse funktsiî,” Matematički Vesnik, vol. 39, no. 4, pp. 405-415, 1987.

[2] S. Tikhonov, "Characteristics of Besov-Nikol'skil class of function," Electronic Transactions on Numerical Analysis, vol. 19, pp. 94-104, 2005.
[3] O. V. Besov, V. P. Il'in, and S. M. Nikol'skiü, Integral Representations of Functions and Imbedding Theorems, vol. 2 of Scripta Series in Mathematics, V. H. Winston \& Sons, Washington, DC, USA; Halsted Press [John Wiley \& Sons], New York, NY, USA, 1979, Edited by M. H. Taibleson.

[4] M. K. Potapov, F. M. Berisha, N. S. Berisha, and R. Kadriu, "Some reverse lp-type inequalities involving certain quasi monotone sequences," Mathematical Inequalities and Applications, vol. 18, no. 4, pp. 1245-1252, 2015.

[5] S. Tikhonov, "Trigonometric series with general monotone coefficients," Journal of Mathematical Analysis and Applications, vol. 326, no. 1, pp. 721-735, 2007.

[6] E. Liflyand and S. Tikhonov, "A concept of general monotonicity and applications," Mathematische Nachrichten, vol. 284, no. 8-9, pp. 1083-1098, 2011.

[7] A. A. Konyushkov, "O klassakh lipshitsa," Izvestiya Rossiiskoi Akademii Nauk. Seriya Matematicheskaya, vol. 21, no. 3, pp. 423-448, 1957.

[8] M. K. Potapov and M. Q. Berisha, "Moduli of smoothness and the Fourier coefficients of periodic functions of one variable," Publications de l'Institut Mathématique (Beograd), vol. 26, no. 40, pp. 215-228, 1979.

[9] G. H. Hardy, J. E. Littlewood, and G. Pólya, Inequalities, Cambridge Mathematical Library, Cambridge University Press, Cambridge, UK, 2nd edition, 1988.

[10] E. T. Copson, "Note on series of positive terms," Journal of the London Mathematical Society, vol. 1-3, no. 1, pp. 49-51, 1928.

[11] L. Leindler, "Generalization of inequalities of Hardy and Littlewood," Acta Scientiarum Mathematicarum (Szeged), vol. 31, pp. 279-285, 1970.

[12] L. Leindler, "Power-monotone sequences and Fourier series with positive coefficients," JIPAM Journal of Inequalities in Pure and Applied Mathematics, vol. 1, no. 1, article 1, 10 pages, 2000.

[13] S. Tikhonov and M. Zeltser, "Weak monotonicity concept and its applications," Trends in Mathematics, vol. 63, pp. 357-374, 2014.

[14] A. Zygmund, Trigonometric Series, vol. 2, Cambridge University Press, Cambridge, UK, 1988, Russian translation, Gosudarstv. Izdat. Inostrannoĭ Literatury, Moscow, 1965.

[15] A. F. Timan, Theory of Approximation of Functions of a Real Variable, vol. 34 of International Series of Monographs in Pure and Applied Mathematics, A Pergamon Press Book. The Macmillan Co., New York, NY, USA, 1963, Translated from the Russian by J. Berry, English translation edited and editorial preface by J. Cossar.

[16] M. Q. Berisha and F. M. Berisha, "On monotone Fourier coefficients of a function belonging to Nikol'skiǐ-Besov classes," Mathematica Montisnigri, vol. 10, pp. 5-20, 1999. 


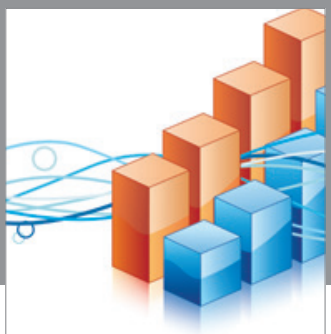

Advances in

Operations Research

vatem alat4

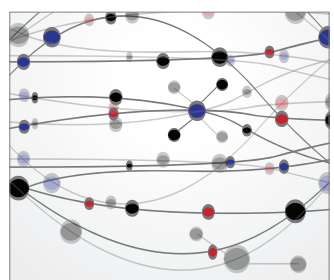

\section{The Scientific} World Journal
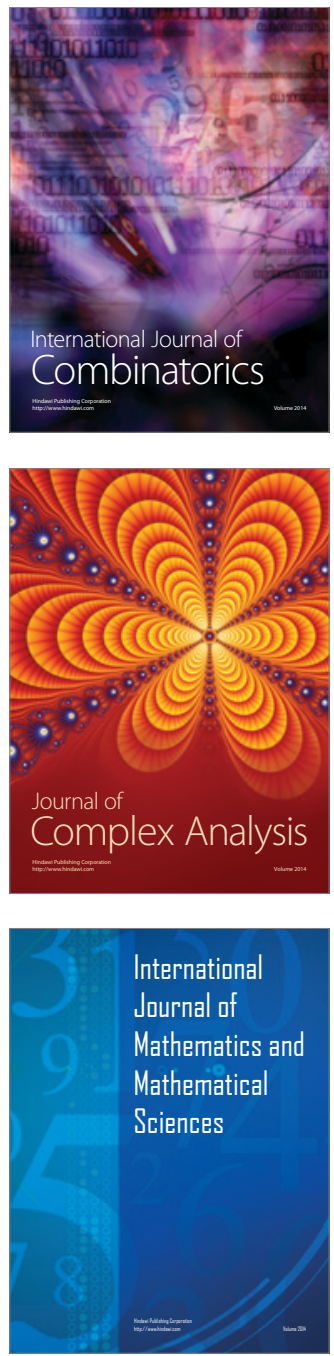
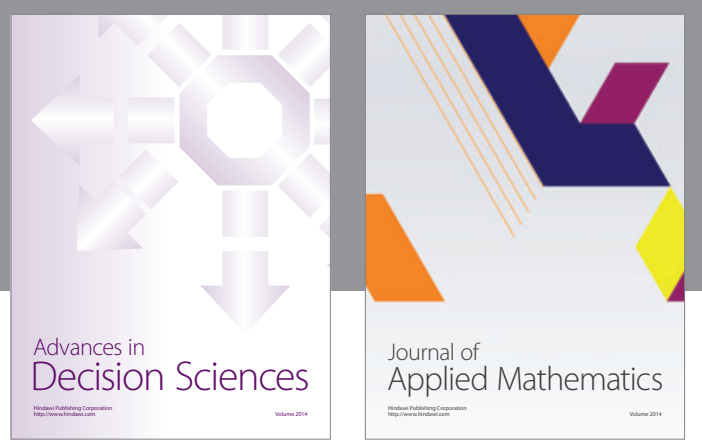

Algebra

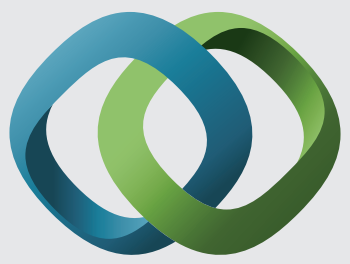

\section{Hindawi}

Submit your manuscripts at

https://www.hindawi.com
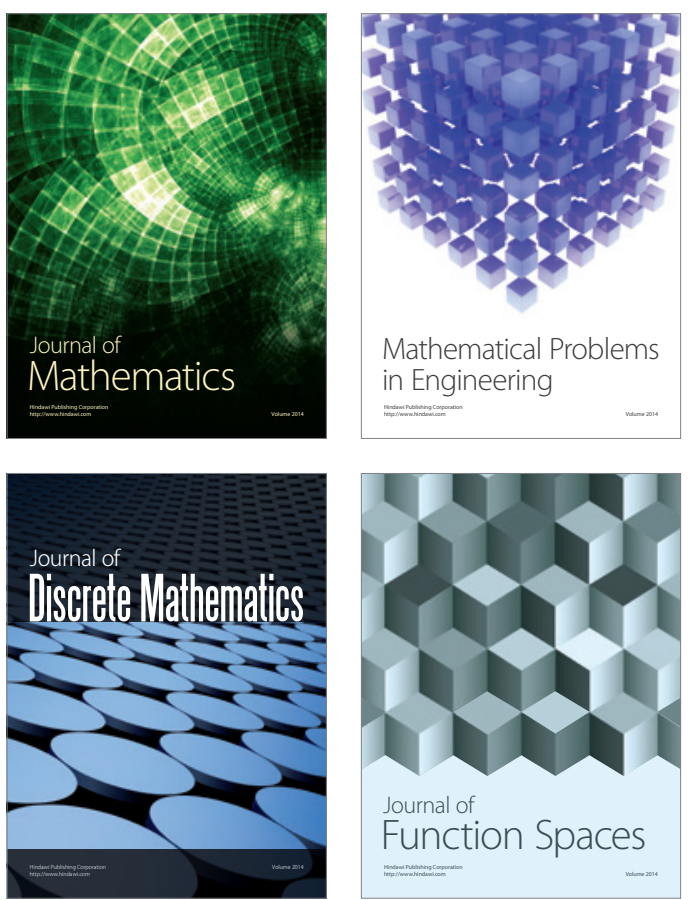

Mathematical Problems in Engineering
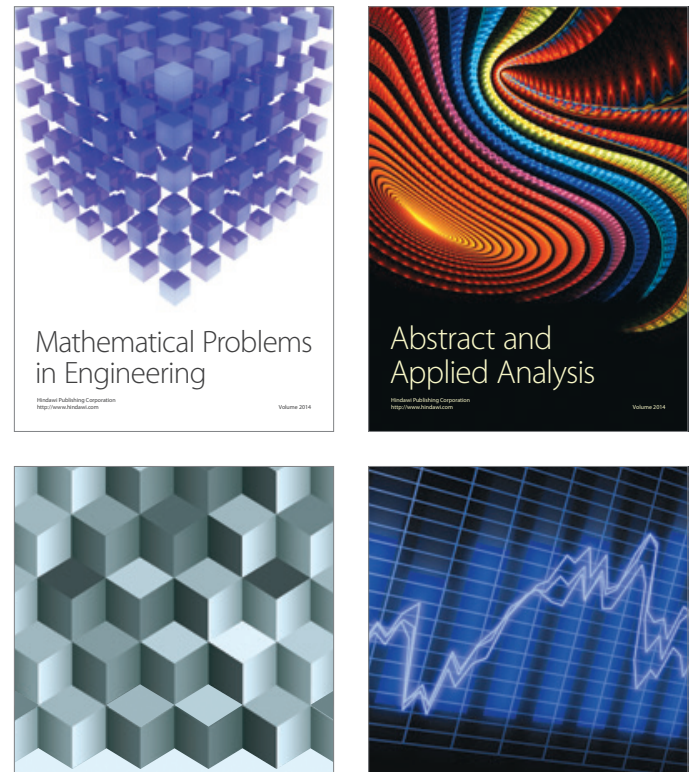

Journal of

Function Spaces

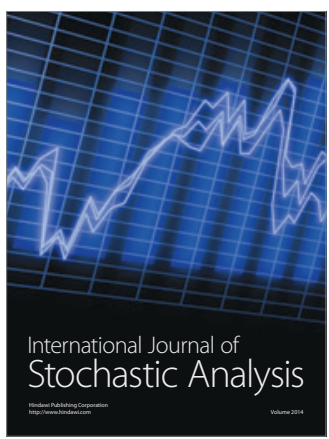

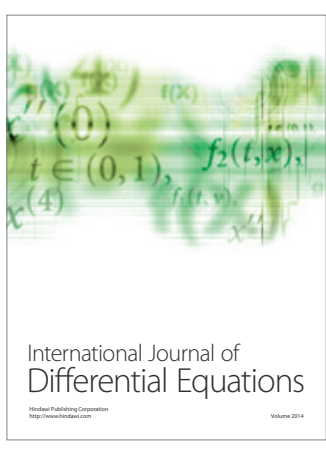
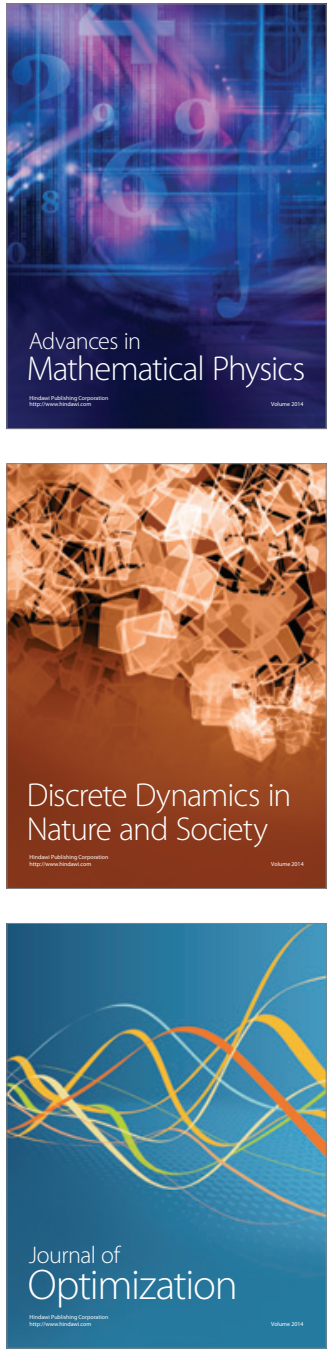